\title{
Repartition Des Glossines Dans La Province De L'ogooue Ivindo Ancien Foyer De Trypanosomose Humaine Africaine
}

\author{
Zinga Koumba Christophe Roland \\ Institut de Recherche en Ecologie Tropicale (IRET), \\ Département de Biologie Animale, Libreville, Gabon \\ Mbang Nguema Ornella \\ Université des Sciences et Techniques de Masuku, \\ Département de Biologie, Franceville, Gabon \\ Midoko Iponga Donald \\ Institut de Recherche en Ecologie Tropicale (IRET), \\ Département de Biologie Végétale; Libreville, Gabon

\section{Mounioko Franck} \\ Université des Sciences et Techniques de Masuku, \\ Département de Biologie, Franceville, Gabon

\section{Mutambwe Shango}

Ecole Régionale Post-Universitaire d'Aménagement et de gestion Intégrés des Forêts et Territoires Tropicaux (ERAIFT), Kinshasa, R. D. Congo

\section{Mavoungou Jacques François}

Institut de Recherche en Ecologie Tropicale (IRET),

Département de Biologie Animale, Libreville, Gabon

\section{M'batchi Bertrand}

Université des Sciences et Techniques de Masuku,

Département de Biologie, Franceville, Gabon

doi: 10.19044/esj.2016.v12n12p281 URL:http://dx.doi.org/10.19044/esj.2016.v12n12p281

\begin{abstract}
In Gabon, the situation of Human African Trypanosomosis (HAT) remains unclear. In addition, several historical foci existing in many provinces have not been explored for over 15 years. Yet many studies have shown that this country has to offer favorable ecosystems for the development of tsetse major vectors of HAT. To verify a risk of transmission of sleeping sickness, a tsetse inventory was conducted in the province of Ogooué Ivindo historic home of HAT. Vavoua and Nzi traps were placed in four characteristic habitats of the province: National Park Ivindo, Zadié Village, Forest of Zadié and baï (clearing) of Momba. A total of 2383 flies
\end{abstract}


were captured: 1680 flies in the clearing of Momba, 437 flies in the primary forest Zadié, 139 in the National Park Ivindo and 12 in Zadié village. Moreover, these flies were divided into 7 species Glossina frezili, Glossina fusca congolensis, Glossina nashi, Glossina palpalis palpalis, Glossina tabaniformis, Glossina fuscipes fuscipes and Glossina tachinoides. Glossina palpalis palpalis (46\%) was the most abundant species followed by Glossina fusca congolensis (21\%) and Glossina nashi (16\%). Glossina fuscipes fuscipes (6\%), Glossina frezili (4\%), Glossina tabaniformis (4\%) and Glossina tachinoides (3\%) were the least caught species. These results have shown that the province of Ogooué Ivindo still remains infested by tsetse flies. Also, further study monitoring these insects is essential to clarify the epidemiological importance of these vectors on human health in this province.

Keywords: Tsetse, vavoua trap, Baï of Momba, National Park of Ivindo, Gabon

\section{Resume}

Au Gabon, la situation de la trypanosomose humaine africaine (THA) demeure encore mal connue. Par ailleurs, plusieurs foyers historiques existent dans de nombreuses provinces et n’ont pas été prospectés depuis plus de 15 ans. Afin de vérifier un risque de transmission de la maladie du sommeil, un inventaire des glossines a été effectué dans la province de l'Ogooué Ivindo, foyer historique de THA. Des pièges Vavoua et Nzi ont été placés dans quatre biotopes caractéristiques de la province, ayant diffèrents degrés de perturbation: parc national de l'Ivindo, village Zadié, Forêt de Zadié et le baï (clairière) de Momba. Au total 2383 glossines ont été capturées dont 1680 dans la clairière de Momba, 437 en forêt primaire de Zadié, 139 dans le parc national de l'Ivindo et 127 au village Zadié. Par ailleurs, ces glossines ont été réparties en 7 espèces dont Glossina frezili, Glossina fusca congolensis, Glossina nashi, Glossina palpalis palpalis, Glossina tabaniformis, Glossina tachinoides et Glossina fuscipes fuscipes. Glossina palpalis palpalis (46\%) a été l'espèce la plus abondante suivie de Glossina fusca congolensis (21\%) et Glossina nashi (16\%). Glossina fuscipes fuscipes (6\%), Glossina frezili (4\%), Glossina tabaniformis (4\%), Glossina tachinoides (3\%) ont été les espèces les moins capturées. Ces résultats ont permis de montrer que la province de l'Ogooué Ivindo demeure toujours infestée par les glossines. Une étude plus approfondie du suivi de ces insectes est indispensable pour préciser l’importance épidémiologique que peuvent avoir ces vecteurs sur la santé humaine des populations de cette province. 
Mots clés : Glossines, pièges vavoua; baï de Momba, parc national de l'Ivindo, Gabon

\section{Introduction}

La maladie du sommeil encore appelée trypanosomose humaine africaine (THA) est endémique dans de nombreux pays d'Afrique Centrale (Kohagne, 2011). Selon l’Organisation Mondiale de la Santé, environ 9878 cas sont notifiés chaque année (OMS, 2015). Ce risque est lié à la fois à la présence de malades qui constituent le principal réservoir de parasites, et au contact avec les glossines ou mouches tsé-tsé (Diptères, Glossinidae) qui sont les seuls vecteurs connus pour la transmission des agents pathogènes responsables de cette parasitose (Gouteux, 2005). Aussi, les populations rurales qui dépendent de la chasse, de l'élevage, de la pêche et de l'agriculture sont les plus exposées aux glossines et par conséquent à la maladie (OMS, 2015). Par ailleurs, cette région d'Afrique Centrale fournit à elle seule $87 \%$ du nombre total de malades diagnostiqués (WHO, 2006 ; Kohagne, 2011). De plus, les maladies à transmission vectorielle figurent parmi les principales causes de morbidité et mortalité dans de nombreux centres hospitaliers (Failloux et al., 1999). Actuellement, ces maladies à transmission vectorielle sont responsables de plus de 17\% des maladies infectieuses et provoquent plus d'un million de décès chaque année (OMS, 2016).

Au Gabon, la situation de la répartition des glossines demeure encore fragmentaire (Zinga et al., 2013a, 2013b). En effet, malgré des travaux conduits par plusieurs auteurs (Kohagne et al., 2010; 2011; Zinga et al., 2013a, 2013b, Zinga et al., 2014 ; Dibakou, et al., 2015, Mbang et al., 2015, Mounioko et al., 2015), il existe peu de connaissances sur la distribution des glossines dans plusieurs localités. Par ailleurs, en raison de ses facteurs bioclimatiques et de la diversité de ses écosystèmes, le Gabon recèle des zones favorables au développement des glossines (Bitome et al., 2015; Dibakou et al., 2015, Mounioko et al. 2015). De plus, le Gabon abrite de nombreux foyers historiques de maladie du sommeil dans certaines régions qui n’ont pas été prospectées depuis plus de 15 ans (Kohagne et al., 2011). Pourtant, des cas sporadiques de THA sont diagnostiqués de manière passive par les structures sanitaires dans ces anciens foyers dont celui de l’Ogooué Ivindo (Kohagne et al., 2010; 2011).

La province de l'Ogooué Ivindo regorge de plusieurs espèces animales dont les éléphants (Loxodonta africana cyclotis), les buffles (Syncerus caffer nanus), les gorilles (Gorilla g gorilla), les chimpanzés (Pan troglodytes) et les céphalophes (Cephalophus spp.) qui sont des hôtes nourriciers des mouches piqueuses (Mavoungou; 2007; Kohagne et al., 2010). La fréquentation régulière du parc national de l‘Ivindo par ces 
animaux fait de cette province un espace écotouristique très prisé par les touristes. Par ailleurs, les contacts entre les populations humaines et animales seront facilités avec la venue importante de touristes mais également avec les populations locales. Aussi, l'une des conséquences possibles de ces contacts sera une augmentation de la probabilité de rencontre hôte-vecteur et donc des échanges de pathogènes entre les humains, la faune et le milieu (Epstein et al., 1993). Finalement, les risques sanitaires vont probablement s'accroître et la fréquence d'apparition de nouveaux cas de parasitose pourrait augmenter au fur et à mesure que les populations humaines seront plus nombreuses à multiplier les contacts avec ces milieux. Ces risques de transmission existent même dans les espaces où la pression humaine est faible, dès lors que les conditions environnementales permettent le contact entre les hôtes infectés et les vecteurs (Fournet et al., 2001).

Dans ce cadre, une étude entomologique basée sur l'inventaire des glossines a été conduite dans la province de l'Ogooué Ivindo pour recueillir des données sur l’abondance et la distribution de ces vecteurs.

\section{Materiel et methodes}

\section{Zone d'étude}

Cette étude a été conduite dans la province de l'Ogooué Ivindo située dans la partie Nord-est du Gabon. Le climat de la région est de type équatorial caractérisé par la double alternance des saisons sèches (de midécembre à mi-mars, puis de mi-juin à mi-septembre) et pluvieuses (de mimars à mi-juin, puis de mi-septembre à mi-décembre) (Mavoungou et al., 2012). La température moyenne est de $24^{\circ} \mathrm{C}$ avec un minimum de $21,7^{\circ} \mathrm{C}$ en juillet et un maximum de $25^{\circ} \mathrm{C}$ en avril. Les amplitudes thermiques annuelles et journalières sont faibles et d'environ $3,3^{\circ} \mathrm{C}$. La pluviométrie annuelle moyenne est de l'ordre de $1700 \mathrm{~mm}$, les plus fortes précipitations ayant lieu en octobre et novembre, puis de mars à mai (Vande, 2006). La faune forestière du Gabon est riche et diversifiée. La province de l'Ogooué Ivindo en détient une part importante avec une faune parmi les mieux répertoriées du Gabon (Mavoungou et al., 2012). Elle est riche entre autre de 128 espèces de mammifères, 424 espèces d'oiseaux, 65 espèces de reptiles et 27 espèces d'amphibiens (Mavoungou et al., 2012, Zinga et al., 2014).

Les sessions de piégeage se sont déroulées dans la clairière de Momba (ou baï de Momba), dans le village Zadié situé à $50 \mathrm{~km}$ de la ville de Makokou (capitale provinciale de l’Ogooué Ivindo), dans la forêt primaire attenant au village Zadié et dans le parc national de l'Ivindo. Dans le parc, les pièges ont été placés le long d'un transect allant de la forêt primaire (FP) au village Loaloa (MA) en passant par la forêt secondaire (FS). 


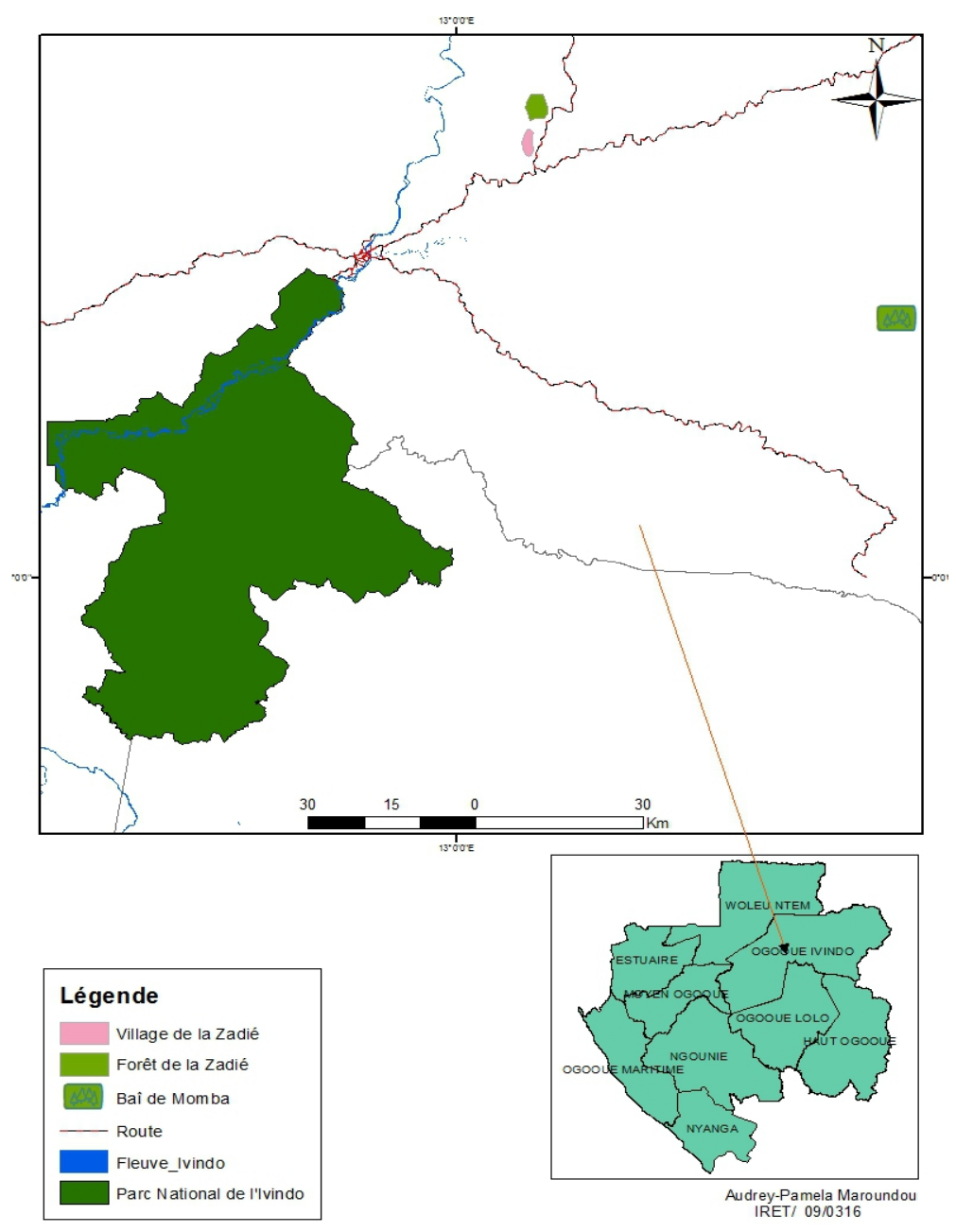

Figure 1 : Localisation de la zone d'étude

\section{Collecte et identification des insectes}

Suivant l'importance des zones prospectées, 10 à 20 pièges Vavoua et Nzi ont été utilisés pour chaque biotope. Les pièges ont été activés le matin avant 7 heures et relevés le soir après 17 heures pendant 20 jours. Lors de la relève des pièges, les cages de capture ont été étiquetées avec le numéro du piège et ramenées au laboratoire pour identification. Cette étude a été effectuée entre janvier et mai 2012.

Au laboratoire, les glossines ont été séparées des autres diptères, puis dénombrées et identifiées sous une loupe binoculaire à l'aide des clefs d'identification publiées par Pollock et Brunhes et al. (1998). La forme des forcipules inférieures nous a permis de distinguer Glossina palpalis palpalis de Glossina fuscipes fuscipes. 


\section{Analyse des données}

L’abondance des espèces de glossines capturées dans la zone d'étude a été définie par leur pourcentage.

L’indice de diversité de Margalef a été calculé pour évaluer la diversité de ces insectes dans les milieux prospectés. Cet indice a été calculé à l'aide de la formule suivante : $\mathrm{D}=(\mathrm{S}-1) / \log \mathrm{N}$, où “ $\mathrm{S}$ ” est le nombre d'espèces et “ $\mathrm{N}$ ” le nombre total d'individus récoltés (Legendre \& Legendre, 1979).

Le test non paramétrique de Kruskal-Walis a été réalisé pour comparer la distribution des espèces suivant les biotopes prospectés. Par ailleurs, les comparaisons multiples par paires suivant la procédure de SteelDwass-Critchlow-Fligner/Test bilatéral ont été effectuées pour comparer la distribution des différentes espèces entre les biotopes prospectés.

Toutes les analyses ont été effectuées à l'aide du logiciel XLSTAT version 3.01.19349.

\section{Resultats}

\section{Distribution et abondance des Glossinidae en fonction des biotopes prospectés}

Au total 2383 glossines ont été capturées dans les milieux prospectés. Par ailleurs, l'abondance de ces insectes varie suivant les biotopes (Figure 2). En effet, le maximum de capture a été obtenu dans la clairière de Momba (1680 glossines) soit un pourcentage de 70,5\%. Les autres milieux à savoir la forêt primaire de Zadié (437 glossines), le parc national de l'Ivindo (139) et le village Zadié (127 glossines) ont présenté des faibles abondances avec des pourcentages respectifs de $18,3 \%, 5,8 \%$ et $5,3 \%$.

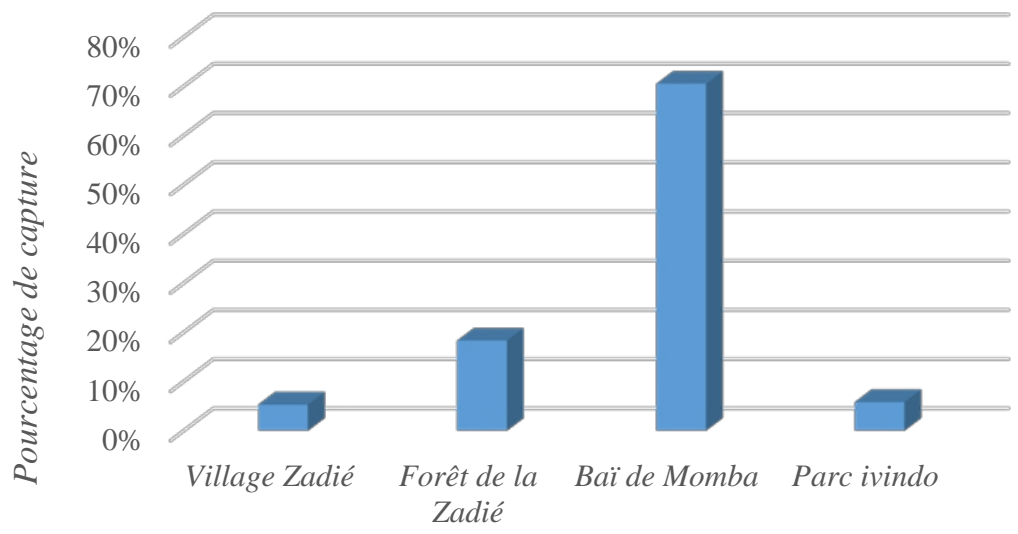

Biotopes prospectés

Figure 2 : Répartition des glossines en fonction des milieux. 


\section{Composition spécifique des glossines capturées dans la zone d'étude}

Au total 7 espèces de glossines ont été capturées dans la zone d'étude avec des abondances variables (Figure 3). Ces espèces ont été Glossina frezili, Glossina fusca congolensis, Glossina nashi, Glossina palpalis palpalis, Glossina tabaniformis, Glossina tachinoides et Glossina fuscipes fuscipes. Elles se repartissent en deux groupes à savoir le groupe Fusca constitué par G. frezili, G. fusca congolensis, G. nashi et G. tabaniformis; et le groupe Palpalis représenté par $G$. palpalis palpalis, G. tachinoides et $G$. fuscipes fuscipes. Le groupe Fusca a représnté 55\% des captures et le groupe Palpalis 45\%.

G. palpalis palpalis (85\%) a été l'espèce la plus abondante du groupe Palpalis. G. fuscipes fuscipes (10\%) et G. tachinoides (5\%) ont été les espèces les moins abondantes dans ce groupe. En ce qui concerne le groupe Fusca, deux espèces ont été abondantes: G. fusca congolensis (45\%) et $G$. nashi (36\%). G. frezili (10\%) et G. tabaniformis (9\%) ont été les deux espèces les moins capturées de ce groupe.

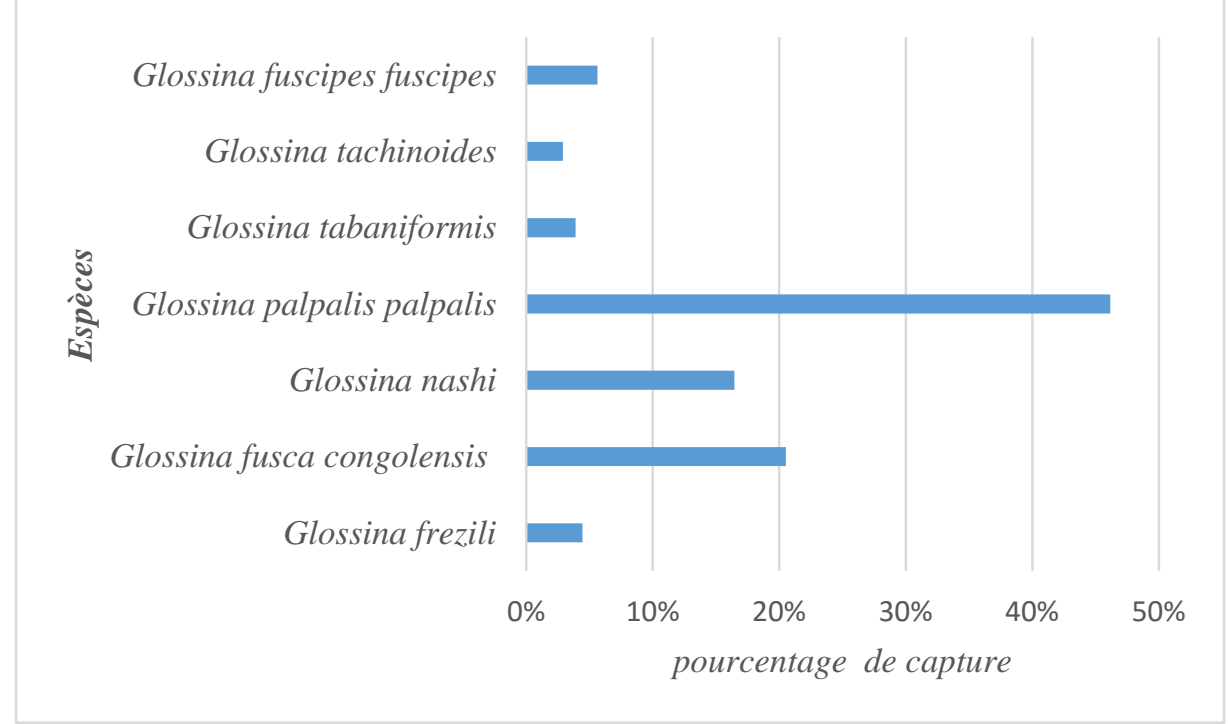

Figure 3 : Répartition des espèces de glossines dans la zone d'étude

\section{Composition et abondance spécifique de glossines capturées en fonction des biotopes}

Au baï de Momba 7 espèces ont été capturées dont $G$. frezili, G. fusca congolensis, G. nashi, G. palpalis palpalis, G. tachinoides et G. fuscipes fuscipes. G. palpalis palpalis (51,7\%) a été l'espèce la plus abondante suivi de $G$. fusca congolensis (23, 3\%) et G. nashi (12,1\%). Quant à G. fuscipes fuscipes (8\%), G. tachinoides (3,4\%), G. frezili (1,3\%) et G. tabaniformis $(0,2 \%)$, elles ont été les espèces les moins capturées. 
Les espèces appartenant au groupe Palpalis (G. palpalis palpalis, G. fuscipes fuscipes et $G$. tachinoides) ont été les plus abondantes dans cette clairière avec un pourcentage total de 63,1\%. Celles du groupe Fusca ont été représentées par G. fusca congolensis, G. nashi, G. tabaniformis et G. frezili soit un pourcentage total $36,9 \%$.

Dans la forêt de Zadié, 5 espèces de glossines ont été identifiées avec des abondances variables. En effet, G. nashi (37\%) et G. palpalis palpalis (26\%) ont été les deux espèces les plus importantes. G. frezili (19\%) et $G$. fusca congolensis (13\%) ont été faiblement capturées. Quant à $G$. tabaniformis (6\%), elle a été l'espèce la moins abondante. Dans ce milieu, le groupe Fusca a constitué $74 \%$ des captures et a été représenté par quatre espèces ( G. frezili, G. tabaniformis, G. nashi, G. fusca congolensis). En ce qui concerne le groupe Palpalis, il n'a été constitué que par G. palpalis palpalis avec un pourcentage de capture de $26 \%$.

Dans le parc national de l'Ivindo G. tabaniformis (46\%) et $G$. palpalis palpalis (32\%) ont été les deux espèces les plus abondantes. Alors que $G$. fusca congolensis (13\%) et $G$. tachinoides (9\%) ont été les espèces les moins représentées. Dans ce parc, les espèces appartenant au groupe Fusca ont été G. tabaniformis et G. fusca congolensis et celles appartenant au groupe Palpalis G. palpalis palpalis et G. tachinoides. Le groupe Fusca a été le plus abondant avec un pourcentage de capture de $59 \%$ contre $41 \%$ pour le groupe Palpalis.

Au village Zadié 4 espèces également ont été identifiées. Ces espèces ont été G. palpalis palpalis, G. Nashi, G. fusca congolensis et G. frezili. G. palpalis palpalis (58\%) a été l'espèce la plus abondante suivi de G. nashi (21\%) et de G. fusca congolensis (19\%). G. frezili (2\%) a été très faiblement capturée. Les espèces du groupe Fusca (G. nashi, G. fusca congolensis et $G$. frezili) ont représenté $42 \%$ des captures alors que $G$. palpalis palpalis, seul représentant du groupe Palpalis, a constitué le maximum de capture dans ce village avec un taux de capture de $58 \%$. 


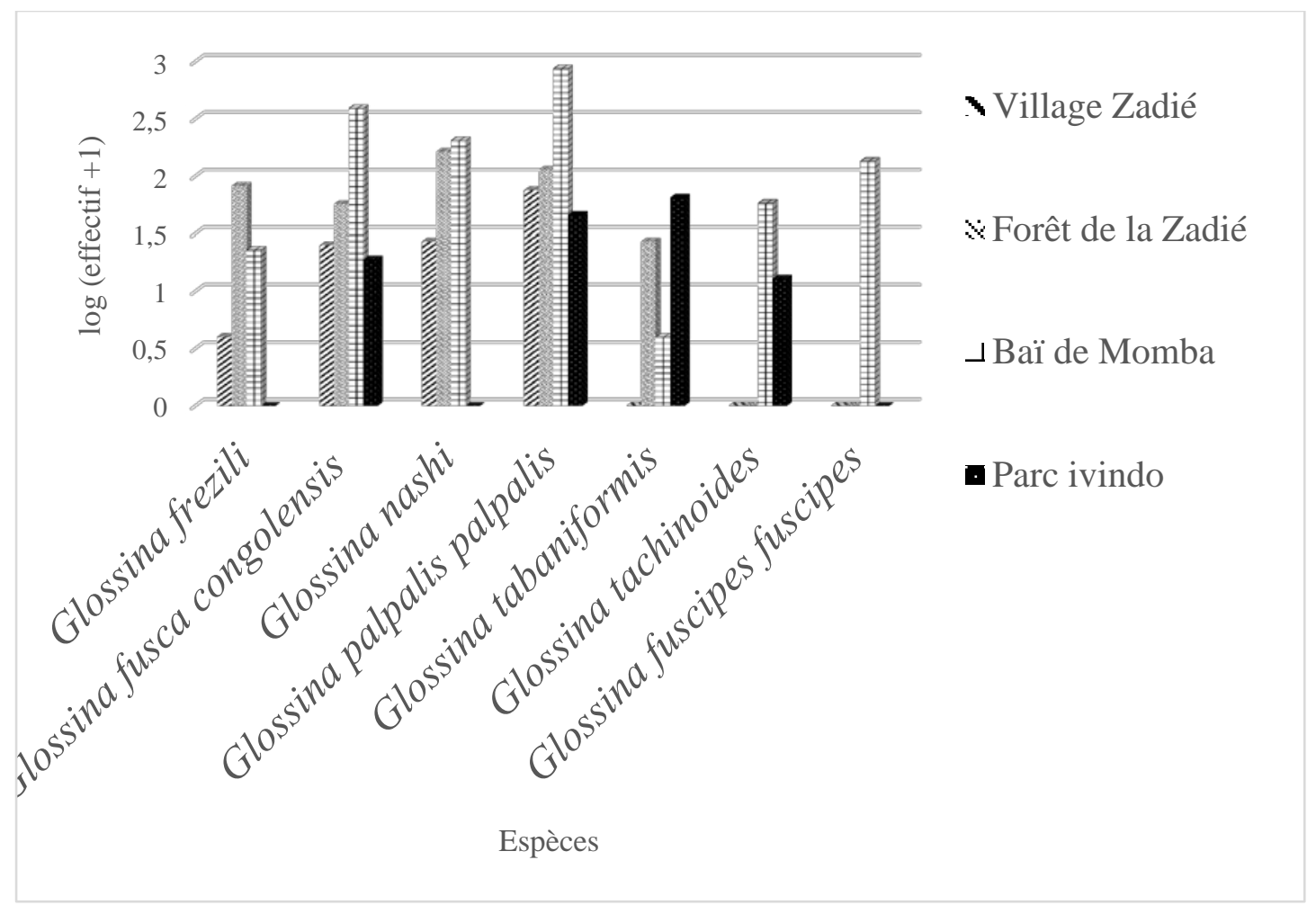

Figure 4 : Distribution spécifique des glossines en fonction des biotopes prospectés.

Le test non paramétrique de Kruskal-Walis a montré qu'il n'existe pas de différence significative dans la distribution des différentes espèces de glossines dans la zone d'étude (Tableau 1). De même, les comparaisons multiples par paires suivant la procédure de Nemenyi ont montré également qu'il n'existe aucune différence significative dans la distribution des espèces de glossines entre les biotopes prospectés (Tableau 2).

Tableau 1 : Résultats du Test de Kruskal-Walis

\begin{tabular}{cc}
\hline K (Valeur observée) & $\mathbf{8 , 0 3 0}$ \\
K (Valeur critique) & 7,815 \\
\hline DDL & 3 \\
\hline p-value (bilatérale) & 0,045 \\
\hline alpha & 0,05 \\
\hline
\end{tabular}

Tableau : Résultats des comparaisons multiples par paires suivant la procédure de SteelDwass-Critchlow-Fligner/Test bilatéral

\section{Différences significatives}

Village Zadié Forêt de la Zadié Baï de Momba Parc Ivindo

\begin{tabular}{cllll}
\hline \multicolumn{2}{c}{ Village Zadié } & Non & Non & Non \\
\hline Forêt de la Zadié & Non & & Non & Non \\
\hline Baï de Momba & Non & Non & & Non \\
\hline Parc Ivindo & Non & Non & Non & \\
\hline
\end{tabular}




\begin{tabular}{ccccc}
\hline \multicolumn{5}{c}{ p-values } \\
\hline & Village Zadié & Forêt de la Zadié & Baï de Momba & Parc Ivindo \\
\hline Village Zadié & $\mathbf{1}$ & 0,475 & 0,128 & 1,000 \\
\hline Forêt de la Zadié & 0,475 & $\mathbf{1}$ & 0,535 & 0,517 \\
\hline Baï de Momba & 0,128 & 0,535 & $\mathbf{1}$ & 0,111 \\
\hline Parc Ivindo & 1,000 & 0,517 & 0,111 & $\mathbf{1}$ \\
\hline
\end{tabular}

\section{Discussion}

Les résultats obtenus dans cette étude constituent des données actualisées relatives à la distribution des glossines dans la province de l'Ogooué Ivindo située au nord-est du Gabon. De même, ces résultats ont permis de confirmer la présence de Glossina palpalis palpalis et Glossina fuscipes fuscipes, vecteurs majeurs de THA dans cette province (Maillot, 1953). Ces résultats confirment aussi l'affinité des espèces du groupe Fusca pour les fôrets et du groupe Palpalis pour les zones riveraines et ripicoles (Djohan et al., 2015). Aussi, ces résultats pourront servir de référence pour des études supplémentaires sur la dynamique spatio-temporelle des glossines dans cette partie du Gabon. Par ailleurs, ces résulats pourront être utilisés dans la mise en place d'un programme de lutte antivectorielle contre ces insectes.

Les pièges Vavoua et Nzi qui ont été utilisés au cours de ce travail ont présenté l'avantage de capturer le groupe cible à savoir les glossines (Laveissière et Grebaut, 1990). Par conséquent, ces résultats confirment l'attractivé de ces pièges dans la capture des mouches hématophages comme l'avaient déjà signalé plusieurs auteurs dont Amsler et Filledier (1994), Gilles et al. (2005), Bitome et al. (2015), Dibakou et al. (2015), Mounioko et al. (2015). Aussi, les résultats obtenus au cours de cette étude pourraient s'expliquer par l'utilisation exclusive de ces deux types de piège. En effet, bien que l'efficacité des pièges Vavoua et Nzi pour la capture des mouches hématophages soit connue (Mihok et al., 1995; Mihok et al., 2002; Gilles, 2005; Gilles et al., 2007; Mavoungou; 2007, Mavoungou et al.; 2012; Zinga et al., 2013a; Zinga et al., 2013b. Mbang et al., 2015), l'association de plusieurs types de piège et l'adjonction d'attractifs olfactifs comme l'octénol seul ou en association avec des phénols auraient permis d'augmenter la taille de l'échantillon (Jaenson et al., 1991 ; Djiteye, 1992; Djiteye ; 1994 ; Mihok et al., 1995; Krčmar et al. 2006; Krčmar 2007; Mihok et al. 2007; Van Hennekeler et al. 2008; Mihok \& Mulye ; 2010; Mihok \& Lange 2012).

L'abondance et la composition des groupes de glossines capturées varient en fonction de leur écologie et des biotopes prospectés. En effet, le groupe Fusca représenté par $G$. frezili, G. fusca congolensis, G. nashi et $G$. tabaniformis a été plus abondant en forêt et au parc national de l'Ivindo. Quant au groupe Palpalis, il a été plus abondant au baï de Momba et au village. Cette répartition pourrait s'expliquer par la bioécologie de chacun 
des groupes capturés. En effet, les glossines du groupe Fusca sont connues pour avoir un tropisme positif pour les zones de forêt et celles du groupe Palpalis pour les zones ripicoles et riveraines (Pollock, 1982, Brunhes et al., 1998). Ces résultats sont similaires à ceux obtenus par Djohan et al.(2015) et Zinga et al. (2013b) qui ont montré une forte abondance du groupe Fusca pour les zones de forêt et du groupe Palpalis pour les zones riveraines et ripicoles.

De même, les espèces de glossines ainsi capturées sont diversement réparties suivant les milieux. En effet, les espèces comme G. palpalis palpalis, G. nashi, G. fuscipes fuscipes, G. tachinoides et $G$. fusca congolensis ont été beaucoup plus nombreuses dans la clairière forestière que dans les trois autres milieux. Quant à G. frezili et G. tabaniformis, elles ont été plus abondantes dans la forêt de Zadié. Cette distribution hétérogène pourrait être liée à la différentiation des paysages et/ou à la structuration de ces biotopes pouvant engendrer des microhabitats particuliers plus ou moins favorables au développement des glossines. Par ailleurs, l'abondance et la richesse spécifique de l'entomofaune en un lieu donné est définie par un certain nombre de facteurs abiotiques et biotiques (Desquesnes et al., 2005).

Les glossines ont été plus abondantes et plus diversifiées dans le baï de Momba que dans les trois autres milieux. Ces résultats seraient en relation avec les exigences écologiques de chacune des espèces capturées en particulier G. palpalis palpalis, G. nashi, G. fuscipes fuscipes, G. tachinoides et $G$. fusca congolensis qui semblent trouver les conditions favorables à leur développement dans cette clairière. Contrairement aux trois autres milieux (parc, village et forêt), la clairière de Momba représente un pôle important de concentration animale pour les grands herbivores tels que les éléphants (Loxodonta africana cyclotis), les buffles (Syncerus caffer nanus), les gorilles (Gorilla gorilla), les potamochères (Potamochoerus porcus) qui y trouvent une végétation dense digestible à croissance rapide, disponible tout le long de l'année (Nganongo, 1999, 2000). Des études conduites par plusieurs auteurs (Frezil \& Carnevale, 1976 ; Laveissière \& Hervouët, 1981 ; Gouteux \& Kiénou, 1982 ; Zinga et al., 2013a, Zinga et al., 2013b ; Zinga et al., 2014) ont montré que la conjugaison de plusieurs facteurs tels qu'une végétation dense et la présence d'hôtes nourriciers (animaux et humains), seraient à l'origine de la forte densité de glossines dans une zone donnée. Aussi, la fréquentation régulière de cette clairière par les animaux semble donc constituer l'un des facteurs déterminant de l'abondance de certaines espèces ( $G$. palpalis palpalis, G. nashi, G. fuscipes fuscipes) dans ce baï.

Par ailleurs, l’indice de diversité de Margalef, qui traduit la richesse spécifique dans un biotope, a été plus important dans le baï de Momba ( $\mathrm{D}=$ $1,86)$, puis en forêt $(D=1,51)$, ensuite au village zadié $(D=1,43)$ et enfin dans le parc. Cependant, les tests non paramétriques de Kruskal-Walis ont montré 
qu’il n’existe pas de différence significative dans la distribution des espèces dans cette région. Il n’existe aucune différence significative dans la distribution de ces insectes entre les milieux.

Les espèces de glossines identifiées dans cette province ont été les espèces aux caractères alimentaires zoophiles (se nourrissant sur les animaux), anthropophiles (se nourrissant sur humains) et même ubiquistes (opportunistes) (Kohagne et al., 2011). Par ailleurs, la présence de $G$. palpalis palpalis et de $G$. fuscipes fuscipes, vecteurs majeurs de la maladie du sommeil, pourrait suggérer l'existence d'un risque sanitaire dans cette zone. De plus, l'ubiquité de G. palpalis palpalis, dans tous les biotopes prospectés, témoigne de sa capacité de résilience et de son affinité pour l'humidité relative et les températures comprise entre 20 et $25^{\circ} \mathrm{C}$, favorisées par la présence des rivières qui constituent, avec la végétation, les facteurs environnementaux favorables pour sa survie (Schwetz, 1915 ; Kohagne et al., 2011). Par ailleurs, la source de nourriture est également l'un des facteurs qui pourrait expliquer la distribution de $G$. palpalis palpalis dont l'éclectisme alimentaire favorise l'adaptation à différents milieux écologiques (Laveissière et al., 2000 ; Kohagne et al., 2011).

L’abondance de $G$. palpalis palpalis dans cette région concorde avec l'ancienne carte de distribution des glossines au Gabon qui a montré une large distribution de cette espèce dans la partie du territoire infestée par ces insectes (Maillot, 1953). Ces résultats corroborent ceux obtenus par Mbida et al. (2009) et Doumba et al. (2016) qui ont montré que les zones à dominance forestière (Ogooué Ivindo) constituent les principaux biotopes favorables au développement des glossines, en particulier $G$. fuscipes fuscipes et $G$. palpalis palpalis qui y trouvent des possibilités de repos et de reproduction.

La présence de G. nashi, G. frezili, G. tachinoides, G. fusca congolensis et $G$. tabaniformis traduirait leur ubiquité et leur affinité pour des zones de forêts et riveraines (Kohagne et al., 2011 ; Dibakou et al., 2015, Dibakou et al., 2015). Ces résultats corroborent ceux obtenus par Mounioko et al. (2015) qui ont montré une forte abondance des glossines dans les forêts présentes dans le parc national de Moukalaba Doudou.

\section{Conclusion}

Les connaissances portant sur les insectes vecteurs d'agent pathogènes, en particulier les glossines, constituent un élément important dans la lutte anti-vectorielle et contre toutes les maladies à transmission vectorielle. Aussi, ce travail s’intègre dans l'étude des insectes vecteurs d'agents pathogènes dans les foyers historiques de la THA au Gabon. Par ailleurs, la problématique des mouches piqueuses est devenue d'actualité dans le cadre de l'impact des changements globaux sur les sociétés humaines, en particulier des maladies émergentes. 
Cette première étude concernant la distribution des glossines dans la province de l'Ogooué Ivindo a permis de mettre en évidence 7 espèces reparties en deux groupes (Palpalis et Fusca) qui vivent en sympatrie dans les différents biotopes prospectés. La province de l'Ogooué Ivindo est une région qui commence à être peuplée surtout avec le développement de la route et de la venue de nombreux touristes. Sachant que le risque de transmission de la maladie du sommeil est d'autant plus grand que la présence humaine dans l'aire de distribution des glossines est durable (Laveissière et al., 1985), on pourrait supposer que les risques de transmission de THA dans cet ancien foyer demeure relativement élevé. De plus, la proximité des humains avec les glossines pourrait dénoter l'existence d'une situation épidémiologiquement dangereuse si le rapprochement entre humains et vecteurs tend à se maintenir (Kohagne, 2011).

Aussi, une étude plus approfondie du suivi de ces insectes est indispensable pour préciser l'importance épidémiologique que peuvent avoir ces vecteurs sur la santé des populations de cet ancien foyer de THA.

\section{Remerciements}

Nous tenons à remercier l'appui financier du projet CoForSet qui a obtenu un financement de la FRB, à travers l'appel à projets 2013 "Scénarios de la biodiversité en Afrique subsaharienne".

Ce travail a également bénéficié de l'appui institutionnel et logistique de l'Institut de Recherche en Ecologie Tropicale (IRET), du Laboratoire d'Ecologie Vectorielle (LEV-IRET), via le projet EU- ACP « Establishment of a Forestry Research network for ACP countries» projet (9 ACP RPR 91 \# 1- FORENET).

\section{References:}

Amblard J. (1996). Situation de la trypanosomiase humaine africaine au Gabon. Etude sur la période 1993-1995. Rapport interne du service des grandes endémies, Ministère de la santé, Gabon, 18p.

Amsler, S., \& Filledier, J. (1994). Attractivité pour les Tabanidae de l'association méta- crésol/octénol. Résultats obtenus au Burkina Faso. Revue d’Élevage et Médecine Vétérinaire des Pays Tropicaux 47, p. 93-96.

Bitome, E.P.Y., Dechaume-Moncharmont, F.X., Mavoungou, J.F.; Mba, O.R., Duvallet, G., \& Bretagnolle F. (2015). Distribution and abundance of hematophagous flies (Glossinidae, Stomoxys, and Tabanidae) in two national parks of Gabon.Parasite22, p. 25.

Brunhes, J., Cuisance, D., Geoffroy; B., \& Hervy J P. (1998). " Les glossines ou Mouches Tsétsé Logiciel d'Identification et d'Enseignement". Editions ORSTOM, Montpellier, France 
Desquesnes, M., Dia, L.M., Acapovi Yao, G.L., Yoni, W., Foil, L., \& Pin, R. (2005). Les vecteurs mécaniques des trypanosomoses animales : Généralités, morphologie, biologie, impacts et contrôle. Identification des espèces les plus abondantes en Afrique de l'Ouest. Bobo-Dioulasso, Burkina Faso, Editions Cirdes, 68 p.

Dibakou, E.S., Mounioko, F., Zinga-Koumba, C.R., Mbang; N.O.A., Acapovi-Yao, G.L., \& Mavoungou, J.F. (2015). Distribution des Glossines vecteurs de la Trypanosomose humaine africaine dans le Parc National de Moukalaba Doudou (Sud-ouest Gabon), Journal of Applied Biosciences86, p. 7957- 7965 .

Djiteye, A. (1992). Aperçu sur l'efficacité comparative de différents pièges et odeurs contre les mouches piqueuses (Diptera : Tabanidae et Muscidae) d’importance vétérinaire. In Premier séminaire International sur les Trypanosomoses Animales non Transmises par les Glossines, Annecy (France), 14-16 octobre.

Djiteye, A. (1994). Efficacité comparée des différents types de pièges et / ou associations d'odeurs sur les tabanidés, stomoxes et glossines présentes en zone soudano-guinéenne (Mali) G. m. submorsitans, G. tachinoides, G. p. gambiensis. Résumé Rapport d’étape, LCV de Bamako, Mali. Krčmar S. (2005). Reponse of horse flies (Diptera, Tabanidae) to different olfactory attractants. Biologica 60, p. 611-613.

Djohan, V., Kaba, D., Rayaissé; JP., Salou, E., Coulibaly, B., Dofini, F., Kouadio K.A.M., Solano, P. (2015). Diversité Spatio-temporelle des glossines le long du Fleuve Comoé en Côte d'Ivoire. Revue d'Elevage et de Médecine Vétérinaire des Pays Tropicaux 68 (1) p.39-44.

Doumba, N.A.G., Zinga, K.C.R., Mounioko, F., Mbang, N.O.A., Mboye, B., Maroundou; A.P., Acapovi-Yao, G.L., M’batchi, B., Mavoungou; J.F. (2015). Composition et diversité spécifique des glossines, vecteurs biologiques de la trypanosomose humaine africaine, dans la région de Ndendé (sud du Gabon).

Epstein, P.R., Ford; T.E., \& Colwell R.R. (1993). Marine ecosystems: emerging diseases as indicators of change. Lancet, 342: 1217-1219.

Failloux, A.B., Vazeille-Falcoz, M., Mousson, L, \& Rodhain F. (1999). Contrôle génétique de la compétence vectorielle des moustiques du genre Aedes. Journée SPE du 13 octobre 1999 (Institut Pasteur à Paris). Société Pathologie et Exotique ; 8pp.

Fournet, F., Kone, A., Meda, A.H., Traore S., \& HERVOUËT, J.P. (2001), Intégration des facteurs démographiques à la caractéristique d'un espace à risque de maladie du sommeil en Côte d’Ivoire. Médecine Tropicale, 61 : 372-375.

Frezil; J.L., \& Carnevale P. (1976). Utilisation de la carboglace pour la capture de Glossina fuscipes quanzensis Pires, 1948, avec le piège Challier- 
Laveissière. Conséquences épidémiologiques. Cahiers ORSTOM, Séries Entomologie Médicale et Parasitologique, 14 : 225-233.

Gilles, J. (2005). Dynamique et génétique des populations d’insectes vecteurs. Les stomoxes, Stomoxys calcitrans et Stomoxys niger niger dans les élevages bovins réunionnais". Thèse de doctorat, Université de La Réunion, St-Denis, 140 pp.

Gilles, J., David, J.F., Duvallet, G., De La Rocque, S., \& Tillard E. (2007). Efficiency of traps for Stomoxys calcitrans and Stomoxys niger on Reunion Island. Medical and Veterinary Entomology 21, p. 65-69.

Gouteux, J.P. (2005). Evaluation du risque dans la maladie du sommeil : apport de la modélisation mathématique. Parasite. 12: 259-264.

Gouteux, J.P., \& Kiénou. (1982). Observations sur les glossines d'un foyer forestier de trypanosomiase humaine en Côte d'Ivoire. Peuplement de quelques biotopes caractéristiques : Plantations, forêts et galeries forestières, en saison des pluies. Cahiers ORSTOM, série Entomologie Médicale et Parasitologie, 20 : 41-61.

Jaenson, G.T., Dos Santos, R.C., \& Hall, D.R. (1991). Attraction of Glossina longipalpalis (Diptera: Glossinidae) in Guinea-Bissau to odorbaited biconical traps. Journal of Medical Entomology28, p. 284-286.

Kohagne, T.L., Gounoue, K.R., Mengue; M.P., Kaba, D., Louis, F.J., \& Mimpfoundi R. (2011). Enquête entomologique dans le foyer historique de trypanosomose Humaine Africaine de Bendjé (Gabon). Parasite 18, p. 1-7p.

Kohagne, T.L., Mengue, M.P., Mimpfoundi, R., \& Louis, F.J. (2010). Régime alimentaire des glossines et diversité des espèces de trypanosomes dans un foyer actif de trypanosomiase humaine africaine au Gabon. Bulletin de la Société de Pathologie Exotique, 103, p 264-271.

Krčmar, S. (2007). Reponse of horse flies (Diptera, Tabanidae) to canopy traps baited with 4-methylphenol, 3-isopropylphenol, and naphthalene. Journal of Vector Ecology 32, p. 188-192.

Krčmar, S., Mikuska, A., \& Merdic, E. (2006). Response of Tabanidae (Diptera) to different naturel attractants. Journal of Vector Ecology 31, p. 262-265.

Laveissière C., \& Hervouët, J. P. (1991). La trypanosomiase humaine en Afrique de l'ouest. Epidémiologie et contrôle. Eds ORSTOM, collection Didactiques.

Laveissière, C., \& Grébaut P. (1990). Recherche sur les pièges à glossines (Diptera : Glossinidae). Mise au point d'un modèle économique : le piège «Vavoua». Tropical Medicine and Parasitology, 41(2): 185-192.

Laveissière, C., Couret, D., Staak, C., \& Hervouët, J.P. (1985). Glossina palpalis et ses hôtes en secteur forestier de Côte d'Ivoire. Relation avec l'épidémiologie de la trypanosomiase humaine. Cahier ORSTOM, série entomologie médicale et parasitologie, 23 (4) : 297-303. 
Laveissière, C., Grébaut, P., Herder, S., \& Penchenier L. (2000). Les glossines vectrices de la Trypanosomiase humaine africaine. IRD and OCEAC, Yaoundé, Cameroun. 246 p.

Maillot, L. (1953). Carte de la distribution probable des sous-espèces et variétés de Glossina palpalis. Échelle 1/28 000 000. ORSTOM, service cartographie, Paris.

Mavoungou, J.F. (2007). Ecologie et rôle vecteur des stomoxes (Diptera : Muscidae) au Gabon. Thèse de doctorat. Université Montpellier III PAUL VALERY, p 137.

Mavoungou, J.F., Makanga, B., Acapovi-Yao, G.L., Desquesnes, M., \& M’batchi B. (2012). Chorologie des Tabanidae (Diptera) dans la réserve de Biosphère Ipassa-Makokou (GABON) en saison des pluies. Parasite 19, p. 165 - 171.

Mbang, N.O.A., Mavoungou, J.F., Mawili-Mboumba, D.P., Zinga-Koumba R.C., Bouyou-Akotet, M.K., \& M'batchi B. (2015). Inventory of potential vectors of trypanosoma and infection rate of the Tsetse fly in the National Park of Ivindo, Gabon. African Health Sciences 15 (3), p. 762-767.

Mbida-Mbida, J.A., Mimpfoundi, R., Njiokou, F., Manga, L., \& Laveissière C. (2009). Distribution et écologie des vecteurs de la trypanosomose humaine africaine de type savanicole en zone de forêt dégradée au sud Cameroun : cas du foyer de Doumé. Bulletin de la Société Pathologique Exotique, 102 : 101-105.

Mihok, S. \& Lange, K. (2012). Synergism between ammonia and phenols for Hybomitra tabanids in northern and temperate Canada. Medical and Veterinary Entomology 26, p. 282-290.

Mihok, S. \& Mulye, H. (2010). Responses of tabanids to Nzi traps baited with octenol, cow urine and phenols in Canada. Medical and Veterinary Entomology 24, p. 266-272.

Mihok, S. (2002). The development of a multipurpose traps (the Nzi) for tsetse and other biting flies. Bulletin of Entomological Research 92, p. 385403.

Mihok, S., Kangethe, E.K., Kamau, G.K. (1995). Trials of Traps and Attractants for Stomoxys spp. (Diptera, Muscidae). Journal of Medical Entomology 32 (3), p. 283-289.

Mounioko, F., Dibakou, F., Zinga-Koumba, C.R., Mbang-Nguema, O.A., Acapovi-Yao, G.L., Mutambwe, S. \& Mavoungou, J.F. (2015). Rythme d’activité journalière de Glossina fuscipes fuscipes, vecteur majeur de la trypanosomiase humaine africaine dans le parc national de Moukalaba Doudou (Sud-Ouest Gabon). International Journal of Biological and Chemical Sciences 9 (1), p. 419-429.

Nganongo, J.B. (1999). Suivi des salines ; Parc national d’Odzala, Congo. Rapports mensuels : Août, septembre, octobre, novembre, décembre. 
Nganongo, J.B. (2000). Suivi des salines ; Parc national d’Odzala, Congo. Rapport mensuel Janvier.

OMS-Organisation Mondiale de la Santé (2015). Maladies à transmission vectorielle. Aide-mémoire N³87, Février 2016

OMS-Organisation Mondiale de la Santé (2015). Trypanosomose humaine africaine (maladie du sommeil). Aide-mémoire N²59, Mai 2015.

Pollock, J N. (1982). "Manuel de lutte contre la mouche Tsé-tsé. Volume 1 : Biologie, systématique et répartition des tsé-tsé. F.A.O; Rome (Italie), 310 p. Schwetz, J. (1915). Preliminary note on the general distribution of Glossina palpalis in Lomani district, Belgian, Congo. Annals of Tropical Medicine and Parasitology, 9: 513-526.

Van Hennekeler, K., Jones, R.E., Skerratt, L.F., Fitzpatrick, L.A., Reid, S.A. \& Bellis, G.A. (2008). A comparison of trapping methods for Tabanidae (Diptera) in North Queensland, Australia. Medical and Veterinary Entomology 22, p. 26-31.

Vande, W J. P. (2006). Ivindo et Mwagna. Eaux noires, foret vièrge et baïs. Wildlife Conservation Society, Libreville (Gabon), 272 p.

WHO-World Health Organization (2006). Weekly epidemiological record. Relevé Epidémiologique hebdomadaire 81 (8), p. 69-80

Zinga-Koumba, C.R., Acapovi-Yao G.L., Mavoungou, J.F., Kohagne, Tongue L., Mbang-Nguema, O.A., Obame, O.P.K., \& Shango; M. (2013a). Influence de la saison sur l'écodistribution des glossines, tabanides, stomoxes du Baï de Momba Makokou, Gabon. Agronomie Africaine 25, p. 149-158.

Zinga-Koumba, C.R., Mbang-Nguema, O.A., Kohagne, T.L., Acapovi-Yao, G.L., Obame, O.K.P, Mutambwe, S., \& Mavoungou, J.F. (2014). Contribution à l'évaluation de la diversité des vecteurs biologiques de la Trypanosomose Humaine Africaine et de leur activité journalière dans le Parc National de l'Ivindo (Nord-est Gabon). Journal of Applied Biosciences 80, p.7060-7070.

Zinga-Koumba, R.C., Bouyer, J., Mavoungou, J.F., Acapovi-Yao, G.L., Kohagne T.L., Mbang-Nguema, O.A., Ondo, O.K.P., \& Mutambwe S. (2013b). Evaluation de la diversité des diptères hématophages dans une clairière marécageuse du Gabon à l'aide des pièges Vavoua et Nzi. Revue d’Elevage et de Médecine Vétérinaire des Pays Tropicaux 66 (3), p.91-96. 